\title{
Extensor tendon ruptures in rheumatoid wrists
}

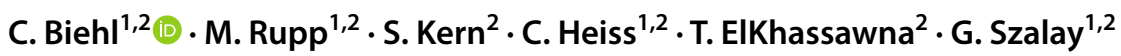

Received: 4 May 2020 / Accepted: 25 June 2020 / Published online: 3 July 2020

(c) The Author(s) 2020

\begin{abstract}
Background and aims Rheumatoid arthritis is a chronic inflammatory disease. The associated involvement of hands and tendons is over $90 \%$ and impairs overall function. In the course of the disease, the joints are often operated on. During this operation, ruptures of the extensor tendons are found by chance without the patients noticing them. The aim of this retrospective study is the prevalence of extensor tendon rupture. Which tendon is destroyed most frequently? How can the functional outcome be measured after reconstruction?

Materials and methods From 1572 operations on rheumatoid wrists, 61 extensor tendon ruptures were identified in 41 patients. The average time between the first rheumatic symptoms of the hand and surgery was 6.4 years. The average duration of RA was 7.8 years. 26 patients with 27 tendon reconstructions were included in the follow-up with an average postoperative duration of 4.6 years ( 3 to 14.2 years).

Results Extensor tendons ruptures typically occurred at mechanically stressed sites. The most frequent rupture was found in the extensor pollicis longus tendon (21 tendons), followed by the small finger extensor tendon (14 tendons). A transfer was performed on 7 tendons. Fifty-five tendon lesions were sutured at other intact tendons. Free grafts were not used. The results in Clayton and QuickDASH scores were significantly different. Functional improvement was consistent with the results of tendon reconstructions in healthy control groups.

Conclusion In rheumatoid patients, a rupture of an extensor tendon must be expected at $4 \%$. Patients tolerate and compensate this damage for a long time. The function of the hand including the tendon function is the most important factor in assessing the success of the operation. The subjective patient acceptance depends on the progress of the underlying disease, postoperative care (ergotherapy, physiotherapy, orthosis) and the patients' demands.
\end{abstract}

Keywords Extensor tendon · Tendon ruptures $\cdot$ Rheumatoid arthritis $\cdot$ Wrist function $\cdot$ Tendon reconstruction $\cdot$ Tendon suture

Electronic supplementary material The online version of this article (https://doi.org/10.1007/s00590-020-02731-1) contains supplementary material, which is available to authorized users.

C. Biehl

Christoph.Biehl@chiru.med.uni-giessen.de

M. Rupp

Markus.Rupp@ukr.de

S. Kern

Stefanie.Kern@chiru.med.uni-giessen.de

C. Heiss

Christian.Heiss@chiru.med.uni-giessen.de

T. ElKhassawna

Thaqif.ElKhassawna@chiru.med.uni-giessen.de

\section{Introduction}

Rheumatoid arthritis (RA) can lead to extensor tendon ruptures. They are not always clinically apparent, especially when peritendinous synovia lead to adhesion of the

\section{G. Szalay}

Gabor.Szalay@chiru.med.uni-giessen.de

1 Klinik Und Poliklinik für Unfall-, Hand- und Wiederherstellungschirurgie-Operative Notaufnahme, UKGM Universitätsklinikum Gießen Und Marburg, Rudolf-Buchheim-Str. 7, 35392 Giessen, Germany

2 Experimentelle Unfallchirurgie, Justus-Liebig-Universität Giessen, Aulweg 128, ForMED (Forschungsgebäude Medizin), 35392 Gießen, Germany 
tendon ends. Malpositioning of the fingers and often painful restrictions of movement are long-term effects. These can lead to ankylosis and affect other fingers and the use of the entire hand [16]. At the same time, many rheumatoid patients become accustomed to the slowly developing limitations (Fig. 1). Statements about the frequency of extensor tendon ruptures at the wrist level (zone VII) are rare. Ehrlich et al. [7] already described individual cases in 1959. Surgeons knew the predilection sites for a tendon rupture at the wrist, the Lister tubercle for the extensor pollicis longus tendon. The destroyed ulna head and the triangular fibrocartilage complex leads to the destruction of the tendon of extensor digiti minimi and extensor carpi ulnae (Fig. 1). Williamson et al. [21] found a prevalence of $1.6 \%$ of ruptured EDM tendon in rheumatoid wrists. Moore et al. [11], Nakamura et al. [12], and Sakuma et al. [18] report larger case numbers and most publications show significantly fewer case numbers.

Surgical treatment aims to improve the function of the wrist. The overall function of the hand and fingers depends on the good function of the tendons. A specific score for finger tendons or finger function does not exist. The known scores (Clayton, Funktionsfragebogen Hannover-functional questionnaire Hannover $(\mathrm{FFbH})$, Activity of Daily Life (ADL), QuickDASH) record the overall situation of hand and arm, but do not differentiate further. In the cohort, patients achieve only below-average scores. The reason for this was seen in the accompanying tendon pathology.

Therefore, the specific benefit for tendon reconstruction or individual finger function is difficult to distinguish based on the mentioned scores.

This study aimed to determine the prevalence of extensor tendon ruptures in rheumatoid wrists and to make statements about the outcome after reconstruction.

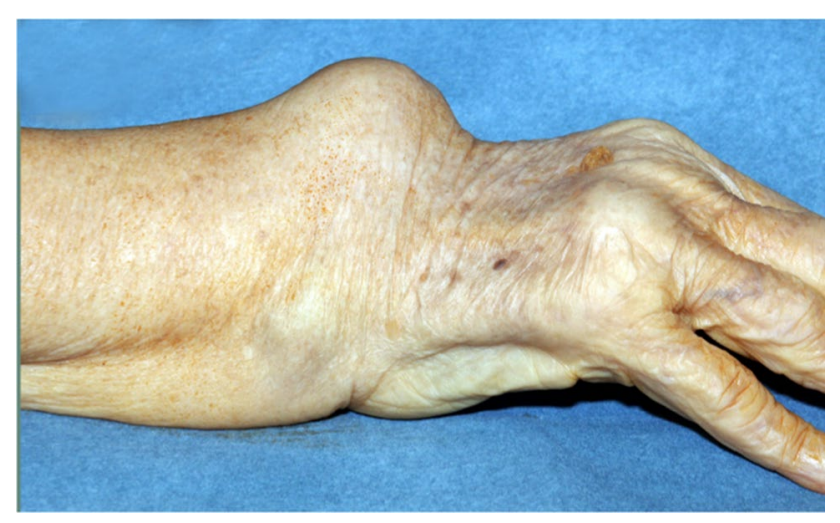

Fig. 1 Clinical aspect of the involvement of extensor tendon in rheumatoid wrist with swelling on the dorsal side and lack of extension in the MCP-joint line

\section{Patients and methods}

This retrospective study includes the treatment of extensor tendon ruptures of the wrist. All patients gave written consent to participate, and ethical approval was not required (Regional Ethics Committee for RhinelandPalatinate; Mainz, Germany).

Between 1984 and 2015, 1572 rheumatoid wrists were surgically treated. An extensor tendon reconstruction was necessary for both acute symptomatic and hidden extensor tendon ruptures during wrist surgery. Postoperatively, the early exercise of the tendons was performed by an ergotherapist. Also, a dynamic therapy with an inverted "Kleinert" extension splint was performed. This causes relief of the tendon suture and at the same time enables a protected movement and had to walk for at least 6 weeks. Afterward, a free extension of the fingers was made possible.

Patients were questioned about their limits in everyday life directly preoperatively and at time of follow-up (12 weeks after the operation) and clinically examined as well. This was monitored using an adjusted score for everyday functions $(\mathrm{FFbH})$, the QuickDASH score, and a 100-point Clayton score (mobility, stability, ligament tension, and pain) $[5,8-10,15,19]$. Only the Clayton Score provides a measuring instrument for the physician. The other scores (FFbH, QuickDASH) are based exclusively on the patient's information. During the follow-up, the authors examined the parameters of pain, strength, range of motion, and everyday functions.

However, due to the small group size and the lack of standard distribution, the statistical evaluation of the nonparametric procedures was performed with the Mann-Whitney $U$ test and Kolmogorov-Smirnov test. The significance level was set at $p \leq 0.05$ for all analyses. ANOVA and Bonferroni correction were used as controls.

\section{Results}

In 41 patients, 62 extensor tendon ruptures at wrist level were detected after synovectomy of the tendon, with necessary tendon suture. The incidence of a tendon rupture in our cohort is $3.9 \%(62 / 1572)$.

The average age of patients at surgery was 62.2 (range, 32-92) years, the average duration of wrist pathology was 6.4 (range, 2-5) years. The average duration of RA was 7.8 years.

Of these extensor tendon reconstructions, 26 patients with 27 tendon surgeries were examined at follow-up. Five patients had already died at the follow-up. Nine patients 
were no longer identifiable or refused further contact with the department. This corresponds to a responder rate of $66 \%$ of surgically treated wrists (27/41). The average follow-up was 4.6 (range, 33-14.2) years postoperatively.

The extensor tendons were pathologically altered with varying frequency. The question is how high the prevalence of tendon comorbidity of ruptured tendons is. A rupture of the extensor pollicis longus tendon (extensor policis longus: EPL) (21 tendons) was diagnosed most, followed by the small finger extensor tendon (extensor digiti minimi: EDM) (14), the ring finger (11), the index finger (6) and the middle finger (5). The extensor indicis (EI) was affected 4 times, a rupture of the extensor carpi ulnaris tendon (ECU) only once (Fig. 2).

Of 62 extensor tendons, we required 7 primary reconstructions and 55 couplings with other tendons (e.g., $\mathrm{EI}>\mathrm{EPL}$ ). If the 4th and 5th tendons were ruptured, they were sutured with other tendons. However, the tendon of the middle finger was used. No free tendon grafts had to be used. No revisions were necessary. Several tendons had to be reconstructed. The risk of concomitant, additional tendon damage with a tendon rupture was $51 \%$.

The Patient-Reported Outcome Measures (PROMs) on the general everyday function postoperative hardly differed from the preoperative values. The authors asked specific hand and finger function according to the scores of ADL/ $\mathrm{FFbH}$ and QuickDASH. Similar to in assessing subjective power. However, the patients rated the tendon function significantly better than the total function of the hand.

The Clayton Score increased from 49.6 points preoperatively to 60.1 points postoperatively (max. 100 points; $p=0.072$ ), which is still a poor result. The Clayton Score differed significantly from QuickDASH in the postoperative values $(p=0.038)$. In the Functional Questionnaire Score, the patients achieved an average of 20 points postoperatively (FFbH, max. 40 points; $p=0.102$ ). In terms of content, QuickDASH and ADL / FFbH ask for parameters regarding the usability of the hand. The questions in both evaluations are $70 \%$ identical. The results of the Functional Questionnaire Score (ADL / FFbH) were

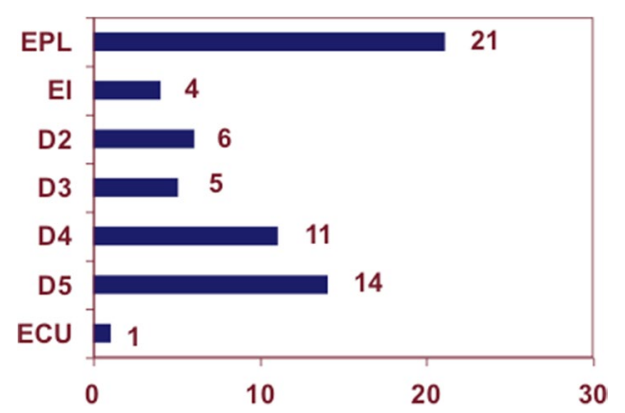

Fig. 2 Extensor tendon ruptures, broken down to finger tendon inversely proportional to the results of the Clayton Scores (no significance, $p=0.72$ ) and QuickDASH $(p \leq 0.01)$.

In nine patients, the scores worsened in the evaluation, in one patient they remained unchanged. Seventeen patients showed better results at follow-up (63\%).

The pain improved from 7.5 points preoperatively on the visual analog scale to 2.9 points postoperatively $(p<0.05)$. The significant decrease in pain is mainly caused by the denervation of the interosseus dorsal nerve. The patients still had limitations and projected the postoperative pain onto the hand as a whole. The authors also asked for impairment of the entire arm. The values show these results (Fig. 3).

The patients did not need revision surgery due to renewed ruptures, adhesions, or infections.

\section{Discussion}

Statements on the incidence of rheumatoid extensor rupture in the literature are rare. Depending on the local situation of the thin periarticular structures, rheumatoid arthritis spreads to the tendons at an early stage and leads to detectable changes $[6,13]$. Patients tolerate tendon pathologies apparently for a long time if they occur slowly and with peritendinous adhesions $[4,20]$. Ruptured tendon sometimes has an artificial function left caused by intact peritendineum. Chung et al. [4] and Barbati et al. [1] describe spontaneous ruptures of damaged extensor tendons as a result of the inflammatory or reactive involvement of the tendons in the disease on the wrist. This matches the experience of the authors.

The primary reconstruction is usually not possible in the ruptured tendon. Publications, therefore, recommend mainly the transfer or coupling of the tendons with an intact extensor tendon [3, 12]. As a second line of defense, many authors favor free transplantation [14, 22]. All tendon operations included in our study were secondary reconstructions with transfer or coupling. A primary suture was never possible, a free graft was not required.

The statistical evaluation in the individual scores revealed poor results. A differentiated evaluation of tendon function was not possible with the scores used. The scores used primarily consider the overall situation of the extremity. Thus, the overall consideration compensated deficits of special care and question. These scores are qualified for patient-related outcome estimation, objective measurements for the affected joint are not possible. There are no finger specific or other scores in the evaluated publications as well.

For this reason, some authors combine different nonspecific scores to make statements on tendon function. Rydholm et al. [17] report in their current publication on testing grip strength in rheumatoid patients. They compared patients' grip strength under basic therapy with 


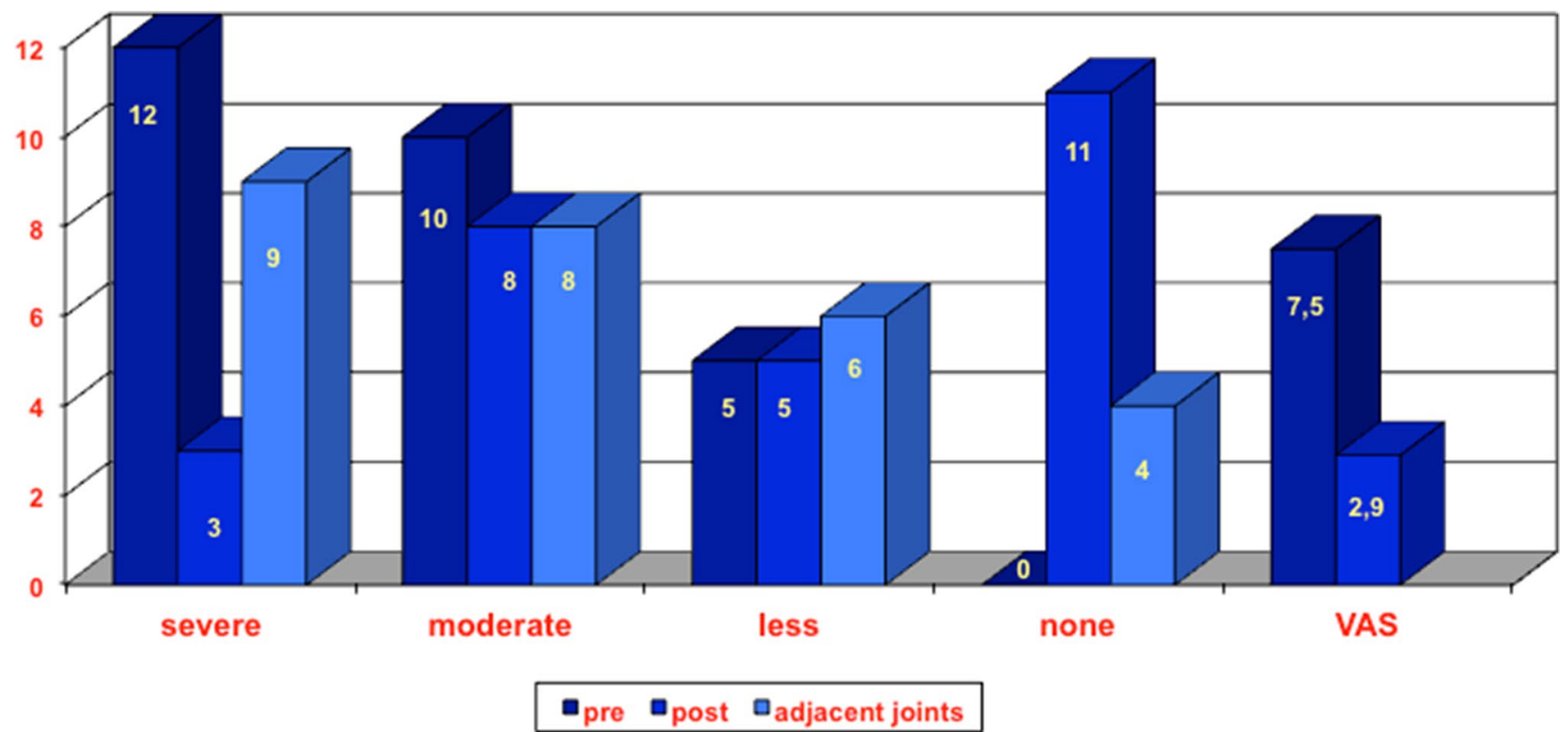

Fig. 3 Patient-reported limits for the wrist pre- and postoperative, adjacent joints of the extremity were evaluated separately (third column). Score results at the visual analog scale

developing the disease activity score (DAS28) of pain on the visual analog scale (VAS) and the Health Assessment Questionnaire (HAQ) [21]. Patients with an inflammatory affection of the wrist or tendons to affect the grip forces were excluded in this study.

In rheumatoid patients, tendon ruptures do not occur in isolation. Depending on the duration of the disease, joints, and tendons of the affected extremity are usually already involved. This leads to poor results. Here the authors have to set the bad initial value in relation.

The patients assessed the overall situation of their hand as sufficient (2.7) and at the same time reported a significant improvement in pain (VAS -4.6 points), when the function of the finger extensors was given. Improvement in the Clayton score (+ 10.5 points) was observed, with wristdependent improvement in the everyday workload. Patient satisfaction depends on finger and extensor tendon function. In most cases, the postoperative stress on the tendons is reduced with less pain.

Rheumatoid patients functionally tolerate and seemingly compensate partial and complete extensor tendon ruptures for a long time. Therefore, contact with the orthopedic surgeon and operative treatment often takes place late. Rydholm et al. [17] report similar results. There is also a demonstrable difference between observed force and subjective patient assessment of the hand function in its publication. In our cohort, the wrists were involved in averaged 6.4 years, before surgical treatment. Other authors report similar results. When assessing the success of surgery, the global function of the wrist is in the foreground. Subjective patient acceptance is consistently good in assessing the tendon. In our cohort, the EPL tendon was most often affected by $21 / 62$ tendon ruptures (34\%). In contrast to this Williamson et al. [21] report, the EDM tendon will rupture first. In our study, only 14/62 tendon ruptures belonged to the EDM tendon (23\%).

Previous studies about the postoperative result showed a strong correlation between good tendon function and patient satisfaction in upper limb surgery [2].

Patients do not see the function of the sewn extensor tendon only on its own but evaluate the suitability for daily use of the entire extremity.

Nevertheless, there are differences between the involved tendons in daily life. Patients with a reconstruction of the

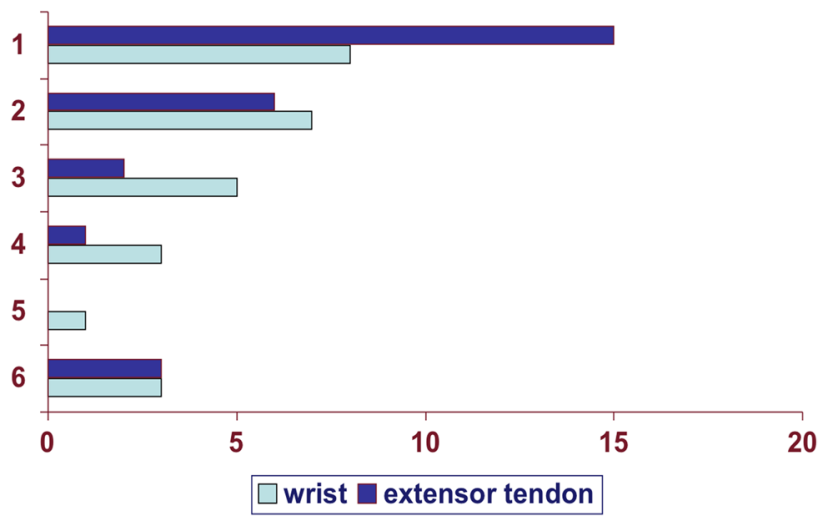

Fig. 4 Subjective patient satisfaction according to school notes $(1=$ best, $6=$ worse $)$ 
EPL tendon still have limitations when using the hand (Fig. 4).

\section{Conclusion}

Rheumatoid patients tolerate and compensate for long term extensor tendon ruptures leading to late clinical presentation. The incidence of an extensor tendon rupture at the wrist is almost $4 \%$ and the prevalence of an additional rupture of another tendon is over 50\%. Advanced tendon rupture allows only the coupling or transfer of non-affected tendons. The functional scores evaluate the overall situation. A specific score for finger function does not exist. However, the global wrist function is crucial for the daily life of patients, which must be taken into account when planning and assessing therapeutic options.

Acknowledgement Open Access funding provided by Projekt DEAL. The authors thank Heiner Thabe, Prof. for the opportunity to give his experience in the medical care of rheumatoid patients in general and in this study. He is and remains a great teacher for us. We are also immensely grateful to Jochen Jung, MD (Diakonie Krankenhaus Bad Kreuznach, kreuznacher diakonie) for his comments on an earlier version of the manuscript or lecture, although any errors are our own and should not tarnish the reputations of this esteemed person.

Author contributions $\mathrm{CB}$ designed the retrospective cohort study and was responsible for data collection. $\mathrm{CB}, \mathrm{MR}$, and GS were responsible for the patient-reported outcomes component of the study. CB, SK, and TE performed the statistical analysis and drafted the manuscript. MR, $\mathrm{TE}, \mathrm{CH}$, and GS corrected the manuscript to improve the quality of written English. GS and TE made substantial contributions to analysis and interpretation of data. All authors read and approved the final manuscript.

Funding There is no source of funding. This research received no specific grant from any funding agency in the public, commercial, or not-for-profit sectors.

\section{Compliance with ethical standards}

Conflicts of interest The authors declare that they have no competing interests. They declare that they have no affiliations with or involvement in any organization or entity with any financial interest or nonfinancial interest in the subject matter or materials discussed in this manuscript.

Data availability and materials The datasets used and/or analyzed during the current study are available from the corresponding author on reasonable request.

Consent for publication Not applicable.

Consent to participate All authors read and approved the final manuscript.

Ethical approval All procedures performed in studies involving human participants were in accordance with the ethical standards of the insti- tutional and/or national research committee and with the 1964 Helsinki declaration and its later amendments or comparable ethical standards. All patients received information about the study and gave written consent. The hospital data protection officer according to the National Institutes of Health guidelines approved the retrospective study design and all investigation protocols. According to the legal bases of the Ethics Committee, State Chamber of Medicine in Mainz, RhinelandPalatinate (chairman Prof. Dr. med. Dipl. Ing. Stephan Letzel; §5a, professional code), no approval was necessary.

Informed consent Due to retrospective nature of the research, no informed consent was requested by the ethical committee. Individual patients cannot be identified.

Open Access This article is licensed under a Creative Commons Attribution 4.0 International License, which permits use, sharing, adaptation, distribution and reproduction in any medium or format, as long as you give appropriate credit to the original author(s) and the source, provide a link to the Creative Commons licence, and indicate if changes were made. The images or other third party material in this article are included in the article's Creative Commons licence, unless indicated otherwise in a credit line to the material. If material is not included in the article's Creative Commons licence and your intended use is not permitted by statutory regulation or exceeds the permitted use, you will need to obtain permission directly from the copyright holder. To view a copy of this licence, visit http://creativecommons .org/licenses/by/4.0/.

\section{References}

1. Barbati A, Geraci A, Borri M et al (2017) Spontaneous rupture of the digital extensor tendons of the hand in unrecognized carpal lunate fracture. Acta Ortop Mex 31:91-94

2. Biehl C, Braun T, Thormann U et al (2018) Radiocarpal fusion and midcarpal resection interposition arthroplasty: long-term results in severely destroyed rheumatoid wrists. BMC musculoskeletal disorders 19:1-7

3. Chu P-J, Lee H-M, Hou Y-T et al (2008) Extensor-tendons reconstruction using autogenous palmaris longus tendon grafting for rheumatoid arthritis patients. J Orthop Surg Res 3:16

4. Chung KC, Kotsis SV (2010) Outcomes of hand surgery in the patient with rheumatoid arthritis. Curr Opin Rheumatol 22:336-341

5. Clayton ML (1965) Surgical treatment at the wrist in rheumatoid arthritis: a review of thirty-seven patients. J Bone Joint Surg Am 47:741-750

6. Doita M, Saura R, Mizuno K (1998) Tendon rupture of the extensor pollicis longus tendon in rheumatoid arthritis. Ryumachi 38:491-495

7. Ehrlich GE, Peterson LT, Sokoloff L et al (1959) Pathogenesis of rupture of extensor tendons at the wrist in rheumatoid arthritis. Arthr Rheumatol 2:332-346

8. Gummesson C, Ward MM, Atroshi I (2006) The shortened disabilities of the arm, shoulder and hand questionnaire (QuickDASH): validity and reliability based on responses within the full-length DASH. BMC Musculoskelet Disord 7:44

9. Lawton MP, Brody EM (1969) Assessment of older people: selfmaintaining and instrumental activities of daily living. Gerontologist 9:179-186

10. Mlinac ME, Feng MC (2016) Assessment of activities of daily living, self-care, and independence. Arch Clin Neuropsychol 31:506-516 
11. Moore JR, Weiland AJ, Valdata L (1987) Tendon ruptures in the rheumatoid hand: analysis of treatment and functional results in 60 patients. J Hand Surg Am 12:9-14

12. Nakamura S, Katsuki M (2002) Tendon grafting for multiple extensor tendon ruptures of fingers in rheumatoid hands. J Hand Surg Br 27:326-328

13. Neurath MF, Stofft E (1993) Ultrastructural causes of rupture of hand tendons in patients with rheumatoid arthritis. a transmission and scanning electron microscopic study. Scand J Plast Reconstr Surg Hand Surg 27:59-65

14. O'sullivan MB, Singh H, Wolf JM (2016) Tendon transfers in the rheumatoid hand for reconstruction. Hand Clin 32:407-416

15. Raspe H, Hagedorn U, Kohlmann T et al (1990/2008) Questionnaire for the diagnosis of functional disability caused by backache. German: Funktionsfragebogen FFbH. Ein Instrument zur Funktionsdiagnostik bei polyartikulären Erkrankungen. In: Sigrist J (Hrsg) Wohnortnahe Betreuung Rheumakranker. In: Rheumatologie DG (ed) Qualitätssicherung in der Rheumatologie. Steinkopff, Stuttgart, New York

16. Rehart S, Sell S, Arbogast M, Aringer M, Arnold I (2015) Expertise Orthopädische Rheumatologie. Georg Thieme Verlag, Stuttgart
17. Rydholm M, Book C, Wikstrom I et al (2018) Course of grip force impairment in patients with early rheumatoid arthritis over the first five years after diagnosis. Arthr Care Res 70:491-498

18. Sakuma Y, Ochi K, Iwamoto T et al (2014) Number of ruptured tendons and surgical delay as prognostic factors for the surgical repair of extensor tendon ruptures in the rheumatoid wrist. J Rheumatol 41:265-269

19. Shauver MJ, Chung KC (2013) The Michigan hand outcomes questionnaire after 15 years of field trial. Plast Reconstr Surg $131: 779 \mathrm{e}-787 \mathrm{e}$

20. VaI L, Vainio KJ (2009) Spontaneous ruptures of tendons in rheumatoid arthritis. Acta Orthop Scand 24:250-257

21. Williamson L, Mowat A, Burge P (2001) Screening for extensor tendon rupture in rheumatoid arthritis. Rheumatology 40:420-423

22. Wilson RL, Devito MC (1996) Extensor tendon problems in rheumatoid arthritis. Hand Clin 12:551-559

Publisher's Note Springer Nature remains neutral with regard to jurisdictional claims in published maps and institutional affiliations. 